\title{
Lung and mediastinum abscesses in Poland: a national hospital register-based study
}

Krzysztof Kanecki ${ }^{1}$, Aneta Nitsch-Osuch ${ }^{1}$, Paweł Goryński², Irena Kosinska ${ }^{1}$, Aleksandra Kozłowska ${ }^{1}$, Katarzyna Okreglicka1 ${ }^{1}$ Piotr Z. Tyszko ${ }^{3}$

\author{
${ }^{1}$ Department of Social Medicine and Public Health, Medical University of Warsaw, \\ Warsaw, Poland \\ ${ }^{2}$ National Institute of Public Health - National Institute of Hygiene, Warsaw, Poland \\ ${ }^{3}$ Institute of Rural Health in Lublin, Lublin, Poland
}

Submitted: 7 July 2019

Accepted: 9 February 2020

Arch Med Sci

DOI: https://doi.org/10.5114/aoms.2020.98741

Copyright (c) 2020 Termedia \& Banach

\begin{abstract}
Introduction: Airway infections represent one of the most common causes of mortality worldwide. The authors sought to describe patients hospitalized for lung and mediastinum abscesses in Poland from 2009 to 2016. To the authors' knowledge, this is the first evaluation of the disease in Poland. Material and methods: The authors conducted a retrospective, populationbased study, using data from 14,932 hospital discharge records. Lung and mediastinum abscesses incidence rates were estimated on the basis of data from a Polish hospital morbidity study, carried out by the National Institute of Public Health.

Results: The study group consisted of 12,049 patients $(74.3 \%$ male and $25.7 \%$ female patients) hospitalized for the first time with the diagnosis of lung or mediastinum abscesses. The mean and median age was 57.2 and 59 years, respectively. The mean age was significantly higher in females than males $(57.9$ years vs. 57 years, $p<0.05$ ). Patients were hospitalized with the following diagnosis: abscess of lung with pneumonia (60.9\%), abscess of lung without pneumonia (24.9\%), abscess of lung and mediastinum $(2.6 \%)$, gangrene and necrosis of lung $(2.8 \%)$, abscess of mediastinum (9.7\%). The average annual incidence of lung abscesses, including gangrene or necrosis of lung, was 35.7 per million ( $95 \% \mathrm{Cl}$ : 34.1-37.4) and the average annual incidence of mediastinum abscess was 4.8 per million $(95 \% \mathrm{Cl}$ : $4.3-5.3)$. The incidence rates for all cases of lung or mediastinum abscesses did not vary significantly between urban and rural regions.

Conclusions: Presented recent data on lung and mediastinum abscesses in Poland may be helpful in comparative analyses among European countries.
\end{abstract}

Key words: hospitalization, pulmonary disease, morbidity dataset.

\section{Introduction}

Lung and mediastinum abscesses are circumscribed collections of pus within the respective parts of the body, which are a result of a microbial infection and may lead to cavity formation. Airway infections have been reported to be one of the most common causes of mortality worldwide. Lung parenchymal abscesses are rare diseases that represent distinct sequelae of a lung infection and may pose a particularly high risk of mortality, if no definitive treatment is applied $[1,2]$. The incidence and mortality
Corresponding author: Krzysztof Kanecki Department of Social Medicine and Public Health Medical University of Warsaw 3 Oczki St 02-097 Warsaw, Poland E-mail: kanecki@mp.pl 
of lung abscesses have decreased significantly over the last few decades [3]. In a study from Poland conducted on a group of 5,151 children hospitalized with pneumonia, 49 complication cases were observed, which constituted $0.95 \%$ of all disease cases. In that study, lung abscess was reported to be a rare and severe pneumonia complication. However, most patients recovered uneventfully without any significant long-term pulmonary sequelae [4]. An increased risk of developing a lung abscess was reported in patients with chronic lung diseases, bronchial obstruction secondary to cancer, a history of aspiration or a higher risk of aspiration caused by alcoholism, altered mental status, physiologic or structural alterations of the pharynx and esophagus, anesthesia and neuromuscular diseases [5]. Although surgical procedures constituted a significant risk for advanced-stage non-small cell lung cancer patients with intratumoral lung abscesses, they led to satisfactory mortality rates and acceptable health prognoses [6]. Other significant predictors of the fatal outcome of surgery for lung abscess are pulmonary sepsis, septic complications, septic organ failure, and preexisting comorbidity [7].

Mediastinal abscess is a rare disease caused by infections involving the mediastinum. It may also occur as a result of a complication arising from cardiac or thoracic surgery after perforation of the aerodigestive tract. In a study from Brazil, 21 patients diagnosed with acute mediastinitis were examined. The study group consisted of 16 males $(76.2 \%)$ and 5 females $(23.8 \%)$. The mean age of participants was 52.5 years, with the range from 23 to 79 years of age. In the study, 6 of the 21 patients died, which resulted in a general mortality rate of $28.6 \%$. No risk factors were found in more than half of the patients. However, $38.1 \%$ of the participants of the study were addicted to smoking, which had been proved in the scientific literature to be a risk factor for mediastinitis. In this study, median sternotomy (38.1\%) and esophageal perforation (33.3\%) were the most common causes of acute mediastinitis [8].

In another study, $14.2 \%$ of patients with lung abscess and sepsis suffered from mediastinitis, the majority of them being women with diabetes mellitus, anemia, obesity and low ejection fraction of the left ventricle [9].

Information on lung and mediastinum abscesses in Poland is based on case reports and reports from selected regions of Poland [4, 10-15]. Hospitalization is widely recommended for diseases that require either numerous or advanced diagnostic or therapeutic procedures. An analysis of hospitalization cases of patients with lung and mediastinum abscesses represents an effective incidence assessment tool. Researchers in the USA [16] and Mexico
[17] have profitably used national administrative databases and patient registries to investigate particular diseases. Implementation of such a scientific approach could be beneficial for research in Poland, too.

Lung and mediastinum abscesses are rare diseases and therefore a large population is required to determine their incidence. There are no current epidemiological data on the burden of lung and mediastinum abscesses in Poland.

The study presented in this paper provides recent data on these diseases on the basis of hospitalization data from national registers in Poland.

\section{Material and methods}

The study is a retrospective population-based study that involved an analysis of hospital discharge records of patients diagnosed with lung or mediastinum abscesses. Data were obtained from the National Institute of Public Health in Poland and covered the period from 2009 to 2016. All hospitals in Poland, except psychiatric and military facilities, are legally obliged to send discharge data to the institute. The data are anonymous and include information on hospitalizations with ICD10-code diagnoses, dates of admission and discharge, date of birth, sex, and place of residence. Two samples were analyzed: 1) all hospitalizations for lung or mediastinum abscesses, and 2) first-time hospitalizations for lung or mediastinum abscesses. In addition, demographic data for the general Polish population were obtained from the Central Statistical Office of Poland [18] Incidence rates were calculated using the number of patients with lung or mediastinum abscesses and corresponding census data. Since lung and mediastinum abscesses are life-threatening diseases, they both require hospitalization. Thus, an analysis of hospitalization cases may be an effective incidence assessment tool. In the study, it was assumed that the hospital diagnosis of lung and mediastinum abscesses is always based on the most current and widely used criteria. In accordance with the Institutional Review Board, no formal consent was required for the study, as it involved a retrospective review covering all patients hospitalized in the years 2009-2016. In the statistical analyses, WINPEPI [19] and Statistica [20] were used. Means, medians, ranges for continuous variables and counts, and percentages for categorical variables were computed. The 95\% confidence intervals (Cls) were estimated, assuming that cases followed a Poisson distribution. Prevalence and incidence rates were calculated by dividing the relevant number of disease cases by the corresponding census figures. Trends were assessed using linear regression. When normality assumptions were not met, non-parametric 
tests ( $\chi^{2}$ test, Mann-Whitney $U$-test) were applied. A two-sided $p$ value below 0.05 was considered to be statistically significant.

\section{Results}

The authors conducted a retrospective, population-based study, using data from 14,932 hospital discharge records. The study group consisted of 12,049 first-time hospitalized patients (74.3\% male and $25.7 \%$ female patients) diagnosed with lung or mediastinum abscess. 423 of them (3.5\%) were patients under 18 years of age. The mean and median age were $57.2(\mathrm{Cl}: 56.9-57.5)$ and 59 years for the first-time hospitalized patients, respectively. The mean age was significantly higher in females than males (57.9 years vs. 57 years, $p<0.05)$. In the subgroup of patients with mediastinum abscess (12.1\% of all patients), there were $29 \%$ females and the mean and median age were 58.8 (Cl: 58-60) and 61, respectively. The age distribution of all participants in the study is presented in Figure 1. Patients were hospitalized with the following diagnoses: abscess of lung with pneumonia $(60.4 \%)$, abscess of lung without pneumonia (24.7\%), abscess of lung and mediastinum (2.5\%), gangrene and necrosis of lung (2.8\%), abscess of mediastinum (9.6\%).

In the study group, the average annual incidence of lung abscesses, including gangrene and necrosis of lung, was estimated at the level of 35.7 per million (95\% Cl: 34.1-37.4), whereas the average annual incidence of mediastinum abscess was 4.8/1,000,000 (95\% Cl: 4.3-5.3). ICD-10 diagnosis and the number of first-time hospitalized and diagnosed patients with per year is presented in Figures 2 and 3. The annual incidence rates of all cases of lung or mediastinum abscesses in the study group were stable over the observation study period 2009-2016. The incidence rates did not differ significantly between urban and rural regions.

In our study, 1392 deaths (12\% of all patients) were observed during the study period (422 females, 970 males; mean age 60 years; min. 19, max. 99 years). 1197 out of 1392 deaths (86\%) were reported in the study group (370 females, 827 males). In the subgroup of patients with mediastinum abscesses, $10 \%$ of patients died during first-time hospitlizations in the study period.

\section{Discussion}

The study showed a significant predominance of males in the group of those diagnosed with lung or mediastinum abscesses. Male predominance was also observed in another study conducted from 1968 to 2004, where lung abscesses were found in 252 consecutive cases of hospitalized patients, 209 of which were observed in males. The mean age in

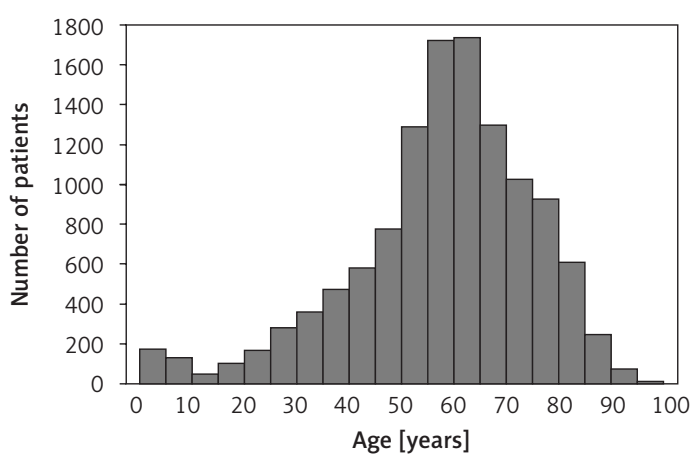

Figure 1. Age distribution of patients at their first hospitalization 2009-2016

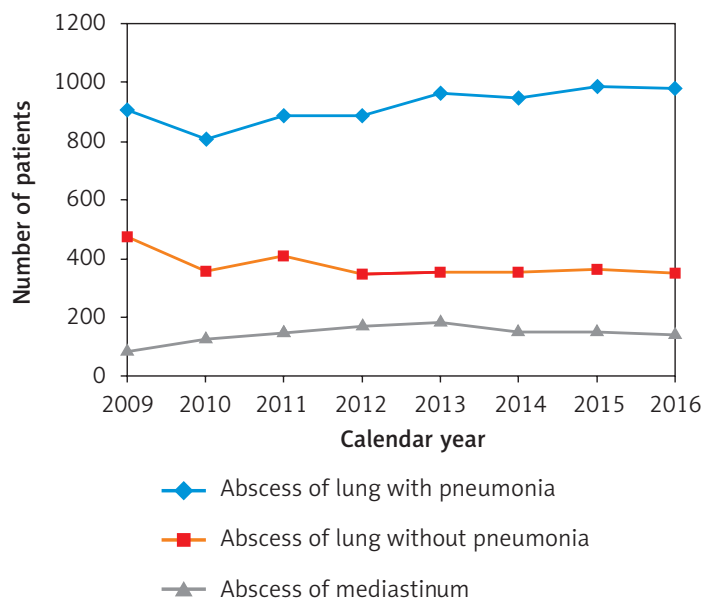

Figure 2. Incidence of first-time diagnosed lung and mediastinum abscesses by year, 2009-2016, part 1

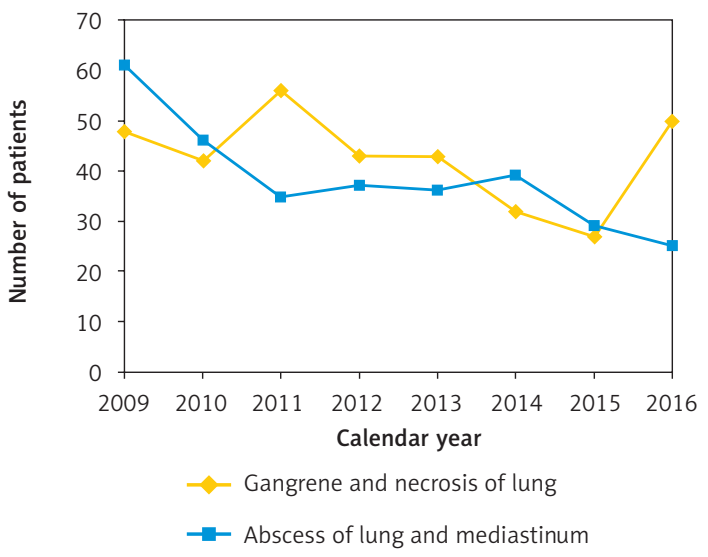

Figure 3. Incidence of first-time diagnosed lung and mediastinum abscesses by year, 2009-2016, part 2

that study was 41.4 years [21]. Another study also showed the predominance of male patients and revealed values similar to our data with the mean age of 56.2 years $( \pm 15.1)$ [22]. The latest study from India reported that the majority of patients with lung abscess were adults, with the mean age of 42.9 years and male to female ratio of $6.6: 1$ [23]. Another report from Madagascar, based on cases 
of pulmonary abscess, showed the mean age of 38 years [24]. Male gender is a factor that can greatly affect the prevalence of the pulmonary abscess. However, it is worth noting that cigarette smoking is a risk factor for the occurrence of diseases that may increase the incidence of lung abscess, such as cardiovascular diseases and periodontal diseases. In a recent multicenter study from Poland, smoking history was reported in $92.6 \%$ of patients with stable chronic obstructive pulmonary disease [25]. Data from a large study on changes in the tobacco prevalence and use in the adult population of Poland in the period from 2003 to 2014 revealed that $30 \%$ of males and $21 \%$ of females were smokers [26]. A high rate of male smokers may indicate a significant influence of cigarette smoking on the occurrence of lung abscesses in Poland. This relationship seems to have been confirmed in other studies. A study based on 111 cases of pulmonary abscess in Madagascar showed that alcohol and tobacco abuse were observed in $32.2 \%$ of disease cases, whereby all patients were exposed to passive smoking [24]. Lack of oral cavity hygiene, abuse of alcohol and smoking were suggested to be causative agents for lung abscess [23]. Additionally, coronary artery bypass graft surgery may be a procedure increasing the risk of mediastinal $a b$ scess. A study from Poland reported the predominance of male patients among patients undergoing coronary artery bypass graft surgery [27].

In our study, patients were hospitalized with abscess of lung and mediastinum (2.5\%) or abscess of mediastinum (9.6\%). In this subgroup, the male to female ratio, mean and median age were similar to those of the study group of all patients. Recent studies have provided limited data on gender and age of patients with mediastinum abscesses.

One study involved an analysis of the data of 34 patients with descending necrotizing mediastinitis for the seven-year period from 2007 to 2013 and showed the mean age of 46.8 years, and the male to female ratio 3.25 [28]. In another study based on the data of 14 patients admitted to the surgical intensive care unit due to acute mediastinitis during the four-year period 20092012, the patients' mean age was 30 years and 8 months, and the male to female ratio was 1.33 [29]. In yet another study based on the data of patients treated surgically for mediastinitis, the outcome was satisfactory in 24 cases and 5 deaths were observed (mortality 17.2\%) [30].

In our study, 423 patients (3.5\%) were under 18 years of age and no deaths were reported. Children with primary or secondary lung abscesses have a significantly better prognosis than adults with the same condition [31]. Additionally, in a study from Poland evaluating risk factors in chil- dren with community-acquired pneumonia, pneumococcal vaccination was reported to be significantly $(p=0.03)$ associated with lower odds ratio for local complications [32].

In our study, 1392 deaths (12\% of all patients) were observed during the study period and $86 \%$ of all deaths were reported in first-time hospitalized patients. All deaths were observed in adults. In another study focusing on patients hospitalized with lung abscess between 1968 and 2004, 4\% of patients died [21]. A study from Madagascar based on 111 cases of pulmonary abscess reported 18 deaths (16.2\%) [24]. Many factors can affect the mortality of patients with pulmonary or mediastinal abscess. Risk factors related to the increased mortality include smoking history and male gender [33]. Old age and associated multiple conditions have also been reported to be related to a worse prognosis [34]. Other negative prognostic factors are senility, neoplasms or bronchial obstruction [35].

The study presented in this paper has several limitations. The database provided the authors with general information on the hospitalization of patients with lung or mediastinum abscesses and to present an epidemiological report. However, the obtained information was not sufficient to examine other risk factors and other correlates of the disease for each patient individually. The diseases in question require treatment or advanced diagnostic procedures over a short period of time, which can be performed in inpatient settings. Importantly, the first diagnosis of lung or mediastinum abscess in the inpatient discharge database might not be the date of the very first diagnosis. This inaccuracy may contribute to an overestimation of the count of incident cases. However, the length of the study observation period may minimize the overestimation.

In conclusion, lung and mediastinum abscesses are rare in Poland and they affect predominantly male patients. In the study presented in this paper, the incidence of lung and mediastinum abscesses did not differ significantly across urban and rural regions. Furthermore, it was stable throughout the observation period. The data obtained in the study may be useful for an analysis of changes in the occurrence of the diseases in Poland, and for comparative studies covering other geographical regions.

\section{Conflicts of interest}

The authors declare no conflict of interest.

\section{References}

1. Kuhajda I, Zarogoulidis K, Tsirgogianni K, et al. Lung abscess-etiology, diagnostic and treatment options. Ann Transl Med 2015; 3:183. 
2. Wali SO. An update on the drainage of pyogenic lung abscesses. Ann Thorac Med 2012; 7: 3-7.

3. Desai H, Agrawal A. Pulmonary emergencies: pneumonia, acute respiratory distress syndrome, lung abscess, and empyema. Med Clin North Am 2012; 96: 1127-48.

4. Wojsyk-Banaszak I, Krenke K, Jończyk-Potoczna K, et al. Long-term sequelae after lung abscess in children - two tertiary centers' experience. J Infect Chemother 2018; 24: 376-82.

5. Gonçalves AM, Menezes Falcão L, Ravara L. Pulmonary abcess, a revision. Rev Port Pneumol 2008; 14: 141-9 [Article in Portuguese].

6. Yamanashi K, Okumura N, Takahashi A, Nakashima T, Matsuoka T. Surgical and survival outcomes of lung cancer patients with intratumoral lung abscesses. J Cardiothorac Surg 2017; 12: 44.

7. Schweigert M, Solymosi N, Dubecz A, et al. Predictors of outcome in modern surgery for lung abscess. Thorac Cardiovasc Surg 2017; 65: 535-41.

8. Fatureto MC, das Neves-Junior MA, de Santana TC. Mediastinite aguda: análise retrospectiva de 21 casos. J Bras Pneumol 2005; 31: 307-11.

9. Shalaeva E, Babadjanov B, Janabaev B, Shalaeva A. Risk factors and incidence of mediastinitis in patients with lung abscess and sepsis. Critical Care. Available at: http://ccforum.biomedcentral.com/articles/10.1186/ cc14011 (Accessed: 23.02.2019).

10. Grzywa-Celińska A, Emeryk-Maksymiuk J, Szmygin-Milanowska K, Czekajska-Chehab E, Milanowski J. Pulmonary actinomycosis - the great imitator. Ann Agric Environ Med 2017; 25: 211-2.

11. Urbanowicz T, Buczkowski P, Budniak W, et al. Computer tomography guided transthoracic periaortic abscess needle biopsy in late mediastinitis after heart surgery. Cardiol J 2014; 21: 325-8.

12. Siemińska A. Pulmonary abscesses in material from tuberculosis and lung disease clinics of the Academy of Medicine in Gdansk. Pneumonol Pol 1990; 58: $307-$ 10 [Article in Polish].

13. Roik D, Mosior T, Sopyło B, Małdyk J, Brzewski M. Abscess mimicking lung metastasis in a 10-year-old boy - case report. Pol J Radiol 2010; 75: 55-57.

14. Lewandowski B, Pakla P, Wołek W, Jednakiewicz M, Nicpoń J. A fatal case of descending necrotizing mediastinitis as a complication of odontogenic infection. A case report. Kardiochir Torakochir Pol 2014; 3: 324-8.

15. Jabłoński S, Modrzewski W, Rysz J, Machała W, Jabłonowski Z, Kordiak J. Pulmonary abscesses - aetiology and treatment. Ten-year experience of the Department of General and Thoracic Surgery in Lodz, Poland. Arch Med Sci 2006; 2: 47-54.

16. Madhani K, McGrath E, Guglani L. A 10-year retrospective review of pediatric lung abscesses from a single center. Ann Thorac Med 2016; 11: 191-6.

17. Fernández-Plata R, Olmedo-Torres D, Martínez-Briseño D, García-Sancho C, Franco-Marina F, González-Cruz H. Prevalence of severe periodontal disease and its association with respiratory disease in hospitalized adult patients in a tertiary care center. Gac Med Mex 2015; 151: 608-13 [Article in Spanish].

18. Statistics Poland. Available at: http://stat.gov.pl (Accessed: 30.07.2018).

19. Abramson JH. WINPEPI updated: computer programs for epidemiologists, and their teaching potential. Epidemiol Perspect Innovations 2011; 8: 1.
20. TIBCO Software Inc. (2017). Statistica (data analysis software system), version 13. http://statistica.io.

21. da Silva Moreira J, de Jesus Peixoto Camargo, Felicetti JC, Goldenfun PR, Schneider Moreira AL, da Silva Porto N. Lung abscess: analysis of 252 consecutive cases diagnosed between 1968 and 2004. J Bras Pneumol 2006; 32: 136-43.

22. Magalhães L, Valadares D, Oliveira JR, Reis E. Lung abscesses: review of 60 cases. Rev Port Pneumol 2009; 15 : 165-78.

23. Mohapatra MM, Rajaram M, Mallick A. Clinical, radiological and bacteriological profile of lung abscess - an observational hospital based study. Open Access Maced J Med Sci 2018; 6: 1642-6.

24. Rakotoson JL, Rebasy L, Andrianasolo R, Rakotoharivelo $\mathrm{H}$, Rakotomizao JR, Andrianarisoa ACF. Pulmonary abscess: a 111-case series in Madagascar. Med Trop (Mars) 2011; 71: 454-6 [Article in French].

25. Płusa T. Quality of life of patients with chronic obstructive pulmonary disease treated with indacaterol and/or glycopyrronium: a real-world Polish observational study. Arch Med Sci Civil Dis 2018; 3: 202-9.

26. Polakowska M, Kaleta D, Piotrowski W, et al. Tobacco smoking in Poland in the years from 2003 to 2014. Multi-centre National Population Health Examination Survey (WOBASZ). Pol Arch Intern Med 2017; 127: 91-9.

27. Trzeciak P, Karolak W, Gąsior M, Zembala M. In-hospital and long-term outcomes of coronary artery bypass graft surgery in patients $\leq 45$ years of age and older (from the KROK registry). Kardiol Pol 2017; 75: 884-92.

28. Palma DM, Giuliano S, Cracchiolo AN, et al. Clinical features and outcome of patients with descending necrotizing mediastinitis: prospective analysis of 34 cases. Infection 2016; 44: 77-84.

29. Razafimanjato NNM, Ralaizafindraibe TH, Ramarolahy AR, Rajaonera TA, Rakotovao HJL. Acute descending necrotizing mediastinitis: four years of experience at a hospital center in Madagascar. Med Sante Trop 2018; 28: 297-301.

30. Bayarri Lara Cl, Sevilla López S, Sánchez-Palencia Ramos A, et al. Surgical management of descending necrotizing mediastinitis. Cir Esp 2013; 91: 579-83 [Article in Spanish].

31. Patradoon-Ho P, Fitzgerald DA. Lung abscess in children. Paediatr Respir Rev 2007; 8: 77-84.

32. Krenke K, Krawiec M, Kraj G, Peradzynska J, Krauze A, Kulus M. Risk factors for local complications in children with community-acquired pneumonia. Clin Respir J 2018; 12: 253-61.

33. Ghazal S, Kumar A, Shrestha B, Sajid S, Malik M, Rizvi N. Risk factors predicting mortality in patients with lung abscess in a public tertiary care center in Karachi, Pakistan. JNMA J Nepal Med Assoc 2013; 52: 571-5.

34. Monteiro R, Alfaro TM, Correia L, Simão A, Carvalho A, Costa JN. Lung abscess and thoracic empyema: retrospective analysis in an internal medicine department. Acta Med Port 2011; 24 Suppl 2: 229-40 [Article in Portuguese].

35. Marra A, Hillejan L, Ukena D. Management of lung abscess. Zentralbl Chir 2015; 140 Suppl 1: 47-53 [Article in German]. 